\title{
PROPRIÉTÉS ÉLECTRONIQUES DES SEMI-CONDUCTEURS AMORPHES $\left(^{*}\right)$
}

\section{J. FORNAZERO}

\author{
Laboratoire de Physique Electronique, Université de Lyon I, 69621 Villeurbanne, France
}

L'état amorphe du point de vue structural et électrique, conduit à une classification des matériaux non cristallins, permettant de situer les verres semiconducteurs. L'exposé comprend deux parties principales :

1. Les ÉTATS électroniques des structures désordonnées. - Leurs caractéristiques dominantes tiennent essentiellement, contrairement aux cristaux parfaits, à la perte de l'ordre à longue portée. L'aléatoire se substitue au périodique. Les fonctions d'onde de Bloch font place à des ondes distordues localisées ou non. $\mathrm{Ce}$ concept de localisation joint à celui du désordre, conduit à la notion de pseudo-gap, de queue de bande, de seuil et gap de mobilité. La perte des relations Energie-Vecteur d'onde donne à la densité d'états un rôle primordial. Les modèles de structures de bandes les plus répandus actuellement sont ceux de Mott-C.F.O., de Davis-Mott et de Marshall-Owen.

2. Les phénomènes de TRANSPORT électroniques dans les S. C. amorphes.

La conduction en régime continu présente trois contributions dominantes :

a) la conduction de bande, pour les énergies supérieures à $E_{\mathrm{C}}$, dans les états localisés.

$\left(^{*}\right)$ Ce texte est un résumé de Conférence. b) la conduction tunnel thermiquement assistée dans les états localisés, près des seuils de mobilité.

c) la conduction tunnel près du niveau de Fermi (celui-ci pouvant être situé au centre du pseudo-gap ou bien dans une bande d'impuretés ou de défauts).

La conduction en alternatif présente des comportements typiques (loi de puissance de $\omega$ et variations en température).

Il est possible de donner une expression du Pouvoir Thermoélectrique, adaptée, valable même en régime de conduction par sauts (hopping) dans les états localisés.

L'effet Hall, enfin conduit souvent à des interprétations délicates, impliquant souvent la contribution des Petits Polarons.

Pour délimiter le sujet il n'est possible que d'évoquer les phénomènes de porosité, élastorésistance, magnétorésistance, résonance de spins, drift mobilité et autres courants de thermodépolarisation...

En conclusion : l'effet de commutation (Switching) des verres, outre son intérêt propre et ses applications, souligne l'aptitude d'un verre à modifier sa structure atomique et ses charges sous l'effet de champs extérieurs appliqués d'intensités modestes, pouvant provoquer des transitions (reversibles ou non) entre état isolant et état conducteur. 